\title{
Steering bound entangled states: A counterexample to the stronger Peres conjecture
}

\author{
Tobias Moroder, ${ }^{1}$ Oleg Gittsovich,,${ }^{2,3,4}$ Marcus Huber, ${ }^{5,6}$ and Otfried Gühne ${ }^{1}$ \\ ${ }^{1}$ Naturwissenschaftlich-Technische Fakultät, Universität Siegen, Walter-Flex-Str. 3, 57068 Siegen, Germany \\ ${ }^{2}$ Institute of Atomic and Subatomic Physics, TU Wien, Stadionallee 2, 1020 Wien, Austria \\ ${ }^{3}$ Institute for Theoretical Physics, University of Innsbruck, Technikerstr. 25, 6020 Innsbruck, Austria \\ ${ }^{4}$ Institute for Quantum Optics and Quantum Information, \\ Austrian Academy of Sciences, Technikerstr. 21a, 6020 Innsbruck, Austria \\ ${ }^{5}$ Departament de Física, Universitat Autònoma de Barcelona, 08193 Bellaterra, Spain \\ ${ }^{6}$ ICFO-Institut de Ciències Fotòniques, Mediterranean Technology Park, 08860 Castelldefels (Barcelona), Spain
}

\begin{abstract}
Quantum correlations are at the heart of many applications in quantum information science and, at the same time, they form the basis for discussions about genuine quantum effects and their difference to classical physics. On one hand, entanglement theory provides the tools to quantify correlations in information processing and many results have been obtained to discriminate useful entanglement, which can be distilled to a pure form, from bound entanglement, being of limited use in many applications. On the other hand, for discriminating quantum phenomena from their classical counterparts, Schrödinger and Bell introduced the notions of steering and local hidden variable models. We provide a method to generate systematically bound entangled quantum states which can still be used for steering and therefore to rule out local hidden state models. This sheds light on the relations between the various views on quantum correlations and disproves a widespread conjecture known as the stronger Peres conjecture. For practical applications, it implies that even the weakest form of entanglement can be certified in a semi-device independent way.
\end{abstract}

Entanglement denotes quantum correlations which cannot be generated in any local way. While the characterization of entanglement for pure two-particle states is straightforward, the task becomes challenging for noisy or mixed quantum states. Here, even the simple question whether or not a given quantum state is entangled is not easy to decide. Apart from that, it is also difficult to characterize the usefulness of entanglement for the mixed state case. Since many quantum information protocols like quantum teleportation or quantum key distribution work with pure maximally entangled states, one may first distill a mixed noisy state to a pure highly entangled state, but characterizing all possible distillation protocols is not straightforward. In fact, it was already shown in 1998 that there are so-called bound entangled quantum states from which no pure state entanglement can be distilled [1]. This shows some irreversibility in entanglement theory, as these states require pure state entanglement for their generation, but then this entanglement can never be recovered again.

In the following years it turned out that bound entangled states are central to many problems in quantum theory. For instance, it has been shown that entangled states with a positive partial transpose (PPT) are bound entangled, but the question whether all bound entangled states are PPT is, despite of numerous efforts [2 4, undecided. Using bound entangled states, it has been shown that bound information, an analogue to bound entanglement in classical information theory, exists in the multipartite scenario [5. Furthermore, bound entangled states are conjectured to have a small dimensionality of entanglement $[\underline{6}$. Finally, it has surprisingly been shown that the correlations of bound entangled states can be used for distilling a secure quantum key [7, 8, although no pure state

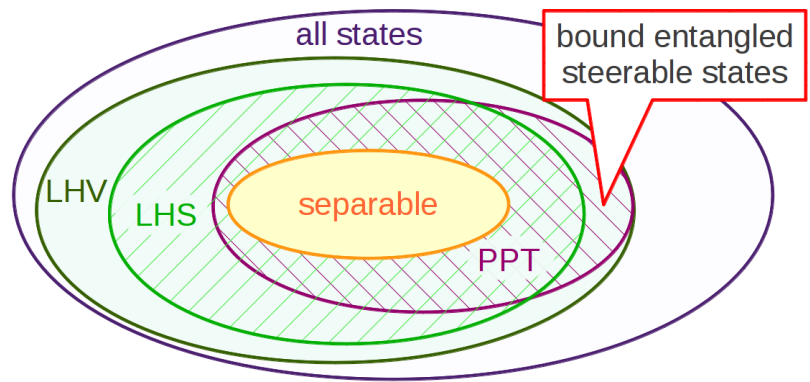

FIG. 1: A schematic view on the space of all quantum states. The set of all states is convex with the separable states as a subset, states which are not separable are entangled. The PPT states are bound entangled, as no pure state entanglement can be distilled from these weakly entangled states. Some states admit a local hidden state (LHS) model, and if this is not the case, they can be used for steering. A larger set of states admits a local hidden variable (LHV) model, and if this is not the case, the state violates some Bell inequality. In this paper we present a method to generate PPT states which are steerable. In this figure we have, according to the Peres conjecture, depicted the PPT states as a subset of the LHV states, but the family of states presented in this paper may also be outside of the LHV states.

entanglement can be distilled from the state. All these problems and observations clearly justify to call bound entanglement a "mysterious invention of nature" [9].

Besides all the applications in information processing, quantum correlations are also important when contradictions between quantum mechanics and the classical world view should be derived. This was highlighted by John Bell, when he showed that no local hidden variable model can reproduce the quantum mechanical correla- 
tions [10, 11]. Interestingly, a similar question was discussed before by Erwin Schrödinger, who asked whether one party (called Alice) can steer the state from the other party (called Bob) by appropriate measurements, a task which is not conceivable in a classical world [12, 13. Mathematically, this problem reduces to finding a local hidden state model for the correlations, which is a hidden variable model with the additional constraint that Bob's measurements are described by the rules of quantum mechanics.

Not surprisingly, bound entanglement is also central to several open problems concerning Bell inequalities and steering, see Fig. 1. Most prominently, a conjecture by Asher Peres ${ }^{1}$ states that bound (and therefore especially PPT) entangled states always admit a local hidden variable model [14]. It is known that this conjecture is wrong in the multipartite case under various different notions of bound entanglement [15, 16, but it is still open in the bipartite case. Here it is known to hold true for various cases [17 23, but it has also been shown that with the help of additional states and operations, any entangled state shows nonlocal behaviour [24, 25]. Similarly, it has been conjectured that all bound entangled states do admit even a hidden state model and are thus useless for steering scenarios [26, 27]. This conjecture is termed the stronger Peres conjecture and recently strong evidence in favor of it has been claimed [27. In this paper we disprove it by giving an explicit counterexample.

More precisely, we present a method to generate systematically bound entangled states which violate a steering inequality and thus do not admit a hidden state model. This does not only deliver the desired counterexample, it also provides candidates for the other conjectures concerning bound entanglement. For instance, these states are natural candidates for testing the original Peres conjecture or the existence of bipartite bound information [5. Finally, the resulting states are interesting from a practical point of view as their entanglement may be verified in experiments without any assumptions on the measurements on one party.

Framework and notation. Steering can be viewed as entanglement verification in a nowadays called semidevice independent scenario [13]. One of the parties, let's say Alice, is totally untrusted and only the number of settings and respective outcomes is specified, while for the other party, Bob, one has a perfect quantum description of the measurements.

We consider the case that Alice can choose between different measurements, each having the same number of possible results. We use $x=1, \ldots, m$ to label the setting, $a=1, \ldots, n$ for the result of the measurement, and $a \mid x$

\footnotetext{
${ }^{1}$ Since the conjecture was suggested by Tal Mor, see Ref. 14], one could even call it Peres-Mor conjecture.
}

for the combination. For Bob we assume that he performs full tomography on his $d$-dimensional system, so that he can reconstruct the state for each possibility $a \mid x$ of Alice. Thus the available data of this scenario are fully specified by the ensemble of conditional states for Bob that we describe by the collection of unnormalized density operators $\mathcal{E}=\left\{\rho_{a \mid x}\right\}_{a, x}$ such that $P(a \mid x)=\operatorname{tr}\left(\rho_{a \mid x}\right)$. Note that non-signalling means that $\sum_{a} \rho_{a \mid x}=\rho$ is independent of the setting $x$, and if this is fulfilled then the ensemble $\mathcal{E}$ indeed has a quantum representation [12, 28.

Note that in a general steering scenario Bob only measures a few characterized observables, e.g., only the Pauli matrices $\sigma_{x}$ and $\sigma_{z}$, or, similarly to Alice, he chooses a setting $y$ and obtains a result $b$ by doing a fixed measurement described by the positive operator valued measure $\left\{M_{b \mid y}\right\}_{b}$. Then the available data are given by the joint conditional probability distributions $P(a, b \mid x, y)$, which admit a local hidden state model if they can be written as

$$
P(a, b \mid x, y)=\sum_{\lambda} P(\lambda) P(a \mid x, \lambda) \operatorname{tr}\left(M_{b \mid y} \sigma_{\lambda}\right) .
$$

Here, $\lambda$ is a hidden variable, occuring with probability $P(\lambda)$ and $\sigma_{\lambda}$ are valid quantum states. In contrast to this, a local hidden variable model would not have such a constraint for Bob's conditional distribution $P(b \mid y, \lambda)$. Note that any distribution, as for instance also $P(a \mid x, \lambda)$ can still always be written as an appropriate measurement on a quantum state 29] - via this one sees that Eq. 11) can be obtained by measuring a separable state. But the important point is that Bob's measurement is fixed.

However, since we assume that Bob obtains full tomography, his exact measurement procedure does not matter. If he obtains full information for instance via separate settings and respective outcomes, then the set of all operators $\left\{M_{b \mid y}\right\}_{b, y}$ spans the full operator space, so that the conditions given by Eq. (1) can only be fulfilled if we have already a corresponding equality on the state space level. Thus, an ensemble $\mathcal{E}$ has a local hidden state model if

$$
\rho_{a \mid x}=P(a \mid x) \frac{\rho_{a \mid x}}{\operatorname{tr}\left(\rho_{a \mid x}\right)}=\sum_{\lambda} P(\lambda) P(a \mid x, \lambda) \sigma_{\lambda}
$$

holds for all choices $a \mid x$. If this is not possible the ensemble $\mathcal{E}$ is called steerable, referring to the phenomena that Alice can steer the decomposition of Bob's reduced state in a non-trivial way.

Before we proceed, note that the problem of Eq. (2) can be simplified if one collects all randomness of $\mathrm{Al}$ ice's measurement into $P(\lambda)$ and Bob's states $\sigma_{\lambda}$. This accounts to consider only the finite number of deterministic strategies for Alice that we label by $\lambda_{i_{1}, i_{2}, \ldots, i_{m}}$ such that the subscripts $i_{k}$ encode the triggered outcome for each setting, i.e., $P\left(a \mid x, \lambda_{i_{1}, \ldots i_{m}}\right)=\delta_{i_{x}, a}$. Then the en- 
semble $\mathcal{E}$ is non-steerable if and only if there exists a set of positive semidefinite operators $\omega_{i_{1} i_{2} \ldots i_{m}} \geq 0$ with $i_{k}=1, \ldots, n$ for each $k=1, \ldots, m$ such that

$$
\rho_{a \mid x}=\sum_{i_{1}, \ldots i_{m}} \delta_{i_{x}, a} \omega_{i_{1} i_{2} \ldots i_{m}}
$$

holds for all possible $a, x$ [26].

All the steering inequalities. Hence to show that an ensemble $\mathcal{E}$ is steerable one must certify that it is not of the form given by Eq. (3). This certificate is called steering inequality and is similar in spirit to Bell inequalities 10, 14, 30 or entanglement witnesses 31. A linear steering inequality is a linear function of the given ensemble $C(\mathcal{E})$ such that $C(\mathcal{E}) \geq 0$ holds for all non-steerable ensembles $\mathcal{E}$, so that $C(\mathcal{E})<0$ witnesses steering.

In order to derive the form of all such steering inequalities one uses Eq. (3) and can proceed along the following lines: The question given Eq. (3) is a special convex optimization problem called semidefinite programming, i.e., $\min _{x \in \mathbb{R}^{n}}\left\{c^{T} x \mid F_{0}+\sum_{i} x_{i} F_{i} \geq 0\right\}$ with $c \in \mathbb{R}^{n}$ and Hermitian matrices $F_{0}$ and all $F_{i}$. Due to the convex structure of the problem one can solve the alternative problem, called dual $\max _{Z \geq 0}\left\{-\operatorname{tr}\left(Z F_{0}\right) \mid \operatorname{tr}\left(Z F_{i}\right)=c_{i}\right\}$, which lower bounds the original problem and finally attains the same optimal value. This dual problem is effectively the optimization over all steering inequalities. Thus to derive all steering inequalities we just need to put Eq. (3) into the form of a semidefinite program and invoke its dual. This approach has been used in a quantification of steering [27].

For our main intended goal we consider only a steering inequality for the case $m=2$ and $n=3$, since this be the setting of our counterexample. It should be noted, however, that our approach can directly be applied also for more than two measurements or more outcomes. In the following we state the form of all such inequalities and verify $C(\mathcal{E}) \geq 0$ for all non-steerable ensembles.

Steering inequality. Consider the described steering scenario for $m=2$ and $n=3$. Suppose we have a set of operators $\mathcal{Z}=\left\{Z_{13}, Z_{23}, Z_{31}, Z_{32}, Z_{33}\right\}$, each positive semidefinite $Z \geq 0$ for all $Z \in \mathcal{Z}$, and further satisfying

$$
\begin{aligned}
& Z_{11}=Z_{13}+Z_{31}-Z_{33} \geq 0 \\
& Z_{21}=Z_{23}+Z_{31}-Z_{33} \geq 0 \\
& Z_{12}=Z_{13}+Z_{32}-Z_{33} \geq 0 \\
& Z_{22}=Z_{23}+Z_{32}-Z_{33} \geq 0
\end{aligned}
$$

Then the linear function

$$
\begin{aligned}
C(\mathcal{E})= & \operatorname{tr}\left(Z_{13} \rho_{1 \mid 1}\right)+\operatorname{tr}\left(Z_{23} \rho_{2 \mid 1}\right)+\operatorname{tr}\left(Z_{31} \rho_{1 \mid 2}\right)+\operatorname{tr}\left(Z_{32} \rho_{2 \mid 2}\right) \\
& +\operatorname{tr}\left[Z_{33}\left(\rho-\rho_{1 \mid 1}-\rho_{2 \mid 1}-\rho_{1 \mid 2}-\rho_{2 \mid 2}\right)\right]
\end{aligned}
$$

is non-negative for all non-steerable ensembles of Eq. (2). Thus $C(\mathcal{E})<0$ shows that the ensemble is steerable.

To show this, note that a given ensemble $\mathcal{E}$ with $m=2$ and $n=3$ is non-steerable if and only if there exists $\omega_{i j} \geq 0$ with $i, j=1,2,3$ such that

$$
\begin{array}{ll}
\rho_{1 \mid 1}=\omega_{11}+\omega_{12}+\omega_{13}, & \rho_{1 \mid 2}=\omega_{11}+\omega_{21}+\omega_{31}, \\
\rho_{2 \mid 1}=\omega_{21}+\omega_{22}+\omega_{23}, & \rho_{2 \mid 2}=\omega_{12}+\omega_{22}+\omega_{32},
\end{array}
$$

and

$$
\rho=\rho_{1 \mid 1}+\rho_{2 \mid 1}+\rho_{3 \mid 1}=\rho_{1 \mid 2}+\rho_{2 \mid 2}+\rho_{3 \mid 2}=\sum_{i j} \omega_{i j}
$$

hold. Using these relations in Eq. 8) one can verify that this expression equals to $C(\mathcal{E})=\sum_{i j} \operatorname{tr}\left(Z_{i j} \omega_{i j}\right)$ and hence is non-negative since all occuring operators are positive semidefinite.

Strategy for generating counterexamples. Now we can present our method of generating bound entangled states. Let us assume that we have fixed a linear steering inequality, i.e., a set of valid operators $\mathcal{Z}$ satisfying the conditions from the previous section. From this one can obtain an entanglement witness 31] by employing any possible choice of measurements for Alice in the expression $C$.

For the case of $n=2, m=3$ this means that the operator

$$
\begin{aligned}
W= & A_{1 \mid 1} \otimes Z_{13}+A_{2 \mid 1} \otimes Z_{23}+A_{1 \mid 2} \otimes Z_{31}+A_{2 \mid 2} \otimes Z_{32} \\
& +\left(\mathbb{1}-A_{1 \mid 1}-A_{2 \mid 1}-A_{1 \mid 2}-A_{2 \mid 2}\right) \otimes Z_{33}
\end{aligned}
$$

is non-negative on separable states for any set of operators $A_{a \mid x}$ satisfying $A_{a \mid x} \geq 0$ and $\sum_{a} A_{a \mid x}=\mathbb{1}$ for all combinations $a, x$ and one readily gets $C=\operatorname{tr}\left(W \rho_{A B}\right)$.

The method is then as follows: We assume that Alice and Bob both have qutrits and that Alice makes a projective measurement in two mutually unbiased bases. After that we look for a "good" steering inequality, i.e., a good set $\mathcal{Z}$. To do so we randomly choose a pure state, compute its ensemble $\mathcal{E}$ using the fixed measurements of Alice, and determine the best steering inequality $\mathcal{Z}$. Afterwards we build up the given witness $W$ and minimize its expectation value with respect to all PPT states. If this optimum is negative, then we have already a counterexample. If this fails then we start over. However, once we found a PPT state violating the randomly chosen steering inequality, we can use this state, compute its ensemble and look for an even better steering inequality. And similarly, once we have a better steering inequality we can look for an even better state. In this sense we further amplify the violation of the PPT entangled state and we repeat this procedure until the violation saturates.

Note that the occurring optimizations are semidefinite programs and thus can be done efficiently [32, 33. Furthermore, we should add that we normalize the steering inequality such that each $Z \in \mathcal{Z}$ satisfies $\operatorname{tr}(Z)=1$.

Counterexample. Running the explained procedure quickly results in bound entangled states which serve as 
counterexamples to the stronger Peres conjecture. Interestingly, if one amplifies the violation, we always end up with a maximal violation of $C=-0.0029$. From the numerical solution one can infer the following analytical solution:

At first let us describe the steering inequality: The set of operators $Z_{13}=\left|q_{+}\right\rangle\left\langle q_{+}\left|, Z_{23}=\right| q_{-}\right\rangle\left\langle q_{-}\right|, Z_{32}=$ $Z_{33}=|s\rangle\langle s|$ and $Z_{31}=(1-x)|t\rangle\langle t|+x| 2\rangle\langle 2|$ with real, normalized vectors

$$
\begin{aligned}
\left|q_{ \pm}\right\rangle & =\left[a, \sqrt{1-a^{2}-b^{2}}, \mp b\right], \\
|s\rangle & =\left[a,-\sqrt{1-a^{2}}, 0\right], \\
|t\rangle & =\left[c,-\sqrt{1-c^{2}}, 0\right]
\end{aligned}
$$

and abbreviations

$$
\begin{gathered}
a=\sqrt{\frac{2}{3}(1+x), \quad b}=\sqrt{\frac{1}{4}(1-2 x)}, \\
c=\sqrt{\frac{2}{3}(1-2 x)} / \sqrt{1-x}
\end{gathered}
$$

defines a one-parameter family of steering inequalities for $0 \leq x \leq 1 / 2$.

This can be seen as follows: With this ansatz we already fulfil the positivity requirements of each individual $Z \in \mathcal{Z}$. Moreover, the additional constraints given by Eqs. (6, 7) are satisfied automatically since $Z_{32}=Z_{33}$, while from Eqs. (4) 5) we only need to check one condition, since the unitary matrix $V=\operatorname{diag}(1,1,-1)$ interchanges $Z_{13}$ with $Z_{23}$, (i.e., $Z_{23}=V Z_{13} V^{\dagger}$ ), but leaves $Z_{31}$ and $Z_{33}$ invariant. Thus we only need to show that $Z_{11} \geq 0$, for which the particular choices of $a, b, c$ become important. These are determined by the identity $Z_{13}+Z_{23}+Z_{31}=\operatorname{diag}(2,1 / 2,1 / 2)$ that we observed from the numerical solution. Via this choices the operator $Z_{11}$ then has the same eigenvalues as $Z_{31}$, i.e., eigenvalues $\{x, 1-x, 0\}$.

Second, before discussing the state, let us fix the two mutually unbiased bases, since we employ here already some rotated form which makes the final bound entangled state look simpler. The respective vectors are denoted by $\left|v_{x \mid a}\right\rangle$ and are given by

$$
\begin{aligned}
& \left|v_{1 \mid 1}\right\rangle=[1 / \sqrt{3},-1 / \sqrt{6},-1 / \sqrt{2}], \\
& \left|v_{2 \mid 1}\right\rangle=[1 / \sqrt{3},-1 / \sqrt{6}, 1 / \sqrt{2}], \\
& \left|v_{3 \mid 1}\right\rangle=[1 / \sqrt{3}, \sqrt{2 / 3}, 0],
\end{aligned}
$$

for the setting $a=1$ and

$$
\begin{aligned}
& \left|v_{1 \mid 2}\right\rangle=[1,0,0], \\
& \left|v_{2 \mid 2}\right\rangle=[0, q / \sqrt{2}, \dot{\mathrm{i}} q / \sqrt{2}], \\
& \left|v_{3 \mid 2}\right\rangle=\left[0, q^{*} / \sqrt{2},-\dot{\mathrm{i}} q^{*} / \sqrt{2}\right],
\end{aligned}
$$

with $q=(-1)^{2 / 3}$ for setting $a=2$.

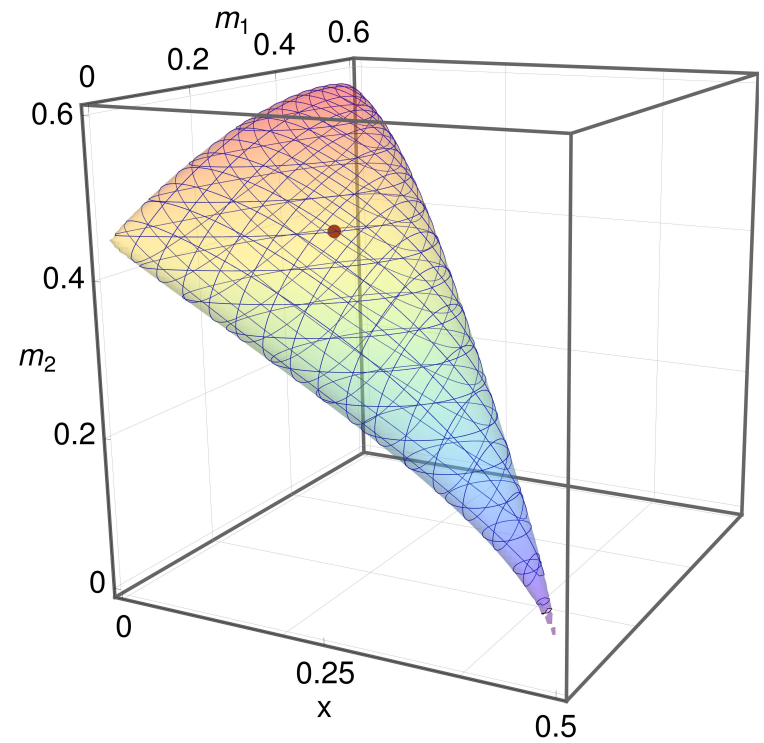

FIG. 2: The family of states which are counterexamples to the stronger Peres conjecture. The parameters $m_{1}$ and $m_{2}$ characterize the state, while $x$ characterizes the steering inequality. The red dot corresponds to the values $x=$ $0.1578, m_{1}=0.2162, m_{2}=0.4363$, resulting in the example given in Eq. 21) and leading to a high violation of the steering inequality.

Finally, let us turn to the state: Consider the following class of states

$$
\begin{aligned}
\rho_{A B} & =\lambda_{1}\left|\psi_{1}\right\rangle\left\langle\psi_{1}\left|+\lambda_{2}\right| \psi_{2}\right\rangle\left\langle\psi_{2}\right| \\
& +\lambda_{3}\left(\left|\psi_{3}\right\rangle\left\langle\psi_{3}|+| \tilde{\psi}_{3}\right\rangle\left\langle\tilde{\psi}_{3}\right|\right)
\end{aligned}
$$

using the normalized states

$$
\begin{aligned}
\left|\psi_{1}\right\rangle & =(|12\rangle+|21\rangle) / \sqrt{2}, \\
\left|\psi_{2}\right\rangle & =(|00\rangle+|11\rangle-|22\rangle) / \sqrt{3}, \\
\left|\psi_{3}\right\rangle & =m_{1}|01\rangle+m_{2}|10\rangle+m_{3}(|11\rangle+|22\rangle), \\
\left|\tilde{\psi}_{3}\right\rangle & =m_{1}|02\rangle-m_{2}|20\rangle+m_{3}(|21\rangle-|12\rangle)
\end{aligned}
$$

with $m_{i} \geq 0$.

By construction, this represents a valid quantum state. In order to assure that this state has a positive partial transpose, we make it PPT invariant, i.e., $\rho_{A B}=\rho_{A B}^{T_{A}}$, for which one must make the off-diagonal blocks Hermitian. These constraints will fix the eigenvalues to

$$
\begin{aligned}
& \lambda_{1}=1-\frac{2+3 m_{1} m_{2}}{4-2 m_{1}^{2}+m_{1} m_{2}-2 m_{2}^{2}}, \\
& \lambda_{3}=\frac{1}{4-2 m_{1}^{2}+m_{1} m_{2}-2 m_{2}^{2}} .
\end{aligned}
$$

The parameter $\lambda_{2}=1-\lambda_{2}-2 \lambda_{3}$ is then given by normalization. The $\lambda_{i}$ are therefore parametrized by $m_{1}, m_{2}$ and this is only giving non-negative eigenvalues 
if $m_{1}^{2}+m_{2}^{2}+m_{1} m_{2} \leq 1$.

Summarizing, we have deduced a class of steering inequalities $\mathcal{Z}$ parametrized by $0 \leq x \leq 1 / 2$, a set of measurements for Alice given by the two mutually unbiased bases, and a class of PPT states that depend on two non-negative, constrained parameters $m_{1}, m_{2}$. For these choices one can now compute expectation values of the steering inequality, and deduce combinations which verify steering, see Fig. 2 .

A simple optimization over these parameters gives
$C=-0.0029$ for $x=0.1578, m_{1}=0.2162, m_{2}=0.4363$.

These parameters then result in vectors

$$
\begin{aligned}
\left|q_{ \pm}\right\rangle & =[0.8785,0.2388, \mp 0.4137] \\
|s\rangle & =[0.8785,-0.4777,0] \\
|t\rangle & =[0.7361,-0.6769,0] .
\end{aligned}
$$

which define the steering inequality and the state

$$
\rho_{A B}=\left[\begin{array}{ccccccccc}
0.026 & 0 & 0 & 0 & 0.0261 & 0 & 0 & 0 & -0.0261 \\
0 & 0.0129 & 0 & 0.0261 & 0.0369 & 0 & 0 & 0 & 0.0369 \\
0 & 0 & 0.0129 & 0 & 0 & -0.0369 & -0.0261 & 0.0369 & 0 \\
0 & 0.0261 & 0 & 0.0526 & 0.0744 & 0 & 0 & 0 & 0.0744 \\
0.0261 & 0.0369 & 0 & 0.0744 & 0.132 & 0 & 0 & 0 & 0.0792 \\
0 & 0 & -0.0369 & 0 & 0 & 0.29 & 0.0744 & 0.0792 & 0 \\
0 & 0 & -0.0261 & 0 & 0 & 0.0744 & 0.0526 & -0.0744 & 0 \\
0 & 0 & 0.0369 & 0 & 0 & 0.0792 & -0.0744 & 0.29 & 0 \\
-0.0261 & 0.0369 & 0 & 0.0744 & 0.0792 & 0 & 0 & 0 & 0.132
\end{array}\right]
$$

That this state is entangled can for instance be checked directly via the covariance matrix criterion [34]. Alternatively, one can even verify that the state does not possess a symmetric extension to two copies of $A$ [35], which is a necessary requirement that the state does not admit a local hidden state model with two settings for $A$ [36]. Note that all numerical values are rounded up to the 4-th digit. One could even take these values explicitly to convince oneself about the counterexample. If one is worried about the numerical precision, then we like to emphasize that all conditions of $\mathcal{Z}$ and $\rho_{A B}$ are easy to check. Using the given 4-digit representation as the actual ones, one can add for instance a small proportion of the identity to form the operators $\tilde{Z}=(1-\epsilon) Z+\epsilon \mathbb{1}$ and the state $\tilde{\rho}_{A B}=(1-\epsilon) \rho_{A B} / \operatorname{tr}\left(\rho_{A B}\right)+\epsilon \mathbb{1} / 9$ such that all constraints are fulfilled and that the smallest eigenvalue is well above the precision. For instance taking $\epsilon=10^{-3}$ amounts that the smallest eigenvalue of all conditions is well above $10^{-5}$ while the violation is still significant $C=-0.0014$.

Conclusion. We provided a way to generate bound entangled states which do not possess a local hidden state model and thus violate a steering inequality. This disproves the stronger Peres conjecture and shows that the original Peres conjecture cannot be proven by considering the stronger steering case. It also means that even the weakest form of entanglement can be verified in a semi-device independent way.

Naturally, the generated bound entangled quantum states are interesting candidates for some of the conjec- tures concerning bound entanglement. A first question is whether with a few further modifications of our states and measurements one could even find a violation of a Bell inequality and thus disprove also the original Peres conjecture. A second question is whether this bound entangled state could even allow the generation of a secret key in a semi-device independent quantum key distribution protocol. Third, these bound entangled states even give prominent candidates to investigate whether they could be useful for teleportation or in entanglement swapping in quantum repeaters [37]. Finally, it would be interesting to use our method to generate bound entangled states in higher dimensions, such as a $4 \otimes 4$ system which be viewed as a four-qubit system. Given the recent advances in quantum control, such states could probably be observed with entangled photons or ions.

We would like to thank J.-D. Bancal, N. Brunner, M. Navascués and Y. C. Liang for stimulating discussions about the Peres conjecture. This work has been supported by the EU (Marie Curie CIG 293993/ENFOQI and Marie Curie IEF 302021/QUACOCOS), the BMBF (Chist-Era Project QUASAR), the FQXi Fund (Silicon Valley Community Foundation), the DFG, the Austrian Science Fund (FWF) and the Marie Curie Actions (Erwin Schrödinger Stipendium J3312-N27).

Note added. After appearance of our results on the arXiv it was noted that if one takes the state from our family with $m_{1}=1 / 60$ and $m_{2}=3 / 10$, the MUB measurements of Alice and the three dichotomic measurements of Bob characterized by the steering inequality, 
more precisely the $Z_{13}, Z_{23}, Z_{33}$ with $x=0.26$, then the corresponding data do not admit a local hidden variable model 38. This shows that our method can indeed be used to find counterexamples to the original Peres conjecture. Note however that this approach is not working for the optimal steering parameters.

[1] M. Horodecki, P. Horodecki, and R. Horodecki, Phys. Rev. Lett. 80, 5239 (1998).

[2] W. Dür, J. I. Cirac, M. Lewenstein, and D. Bruss, Phys. Rev. A 61, 062313 (2000).

[3] P. W. Shor, J. A. Smolin, and B. M. Terhal, Phys. Rev. Lett. 86, 2681 (2001).

[4] J. Watrous, Phys. Rev. Lett. 93, 010502 (2004).

[5] N. Gisin and S. Wolf, Linking Classical and Quantum Key Agreement: Is There Bound Information??, vol. 1880 of Lecture Notes in Computer Science (Springer Berlin Heidelberg, 2000), ISBN 978-3-540-67907-3.

[6] A. Sanpera, D. Bruß, and M. Lewenstein, Phys. Rev. A 63, 050301 (2001).

[7] K. Horodecki, M. Horodecki, P. Horodecki, and J. Oppenheim, Phys. Rev. Lett. 94, 160502 (2005).

[8] K. Horodecki, L. Pankowski, M. Horodecki, and P. Horodecki, IEEE Trans. Inf. Theory 54, 2621 (2008).

[9] R. Horodecki, Europhysics News 41, 21 (2010).

[10] J. S. Bell, Physics 1, 195 (1964).

[11] N. Brunner, D. Cavalcanti, S. Pironio, V. Scarani, and S. Wehner, Rev. Mod. Phys. 86, 419 (2014).

[12] E. Schrödinger, Proc. Camb. Phil. Soc. 31, 555 (1935).

[13] H. M. Wiseman, S. J. Jones, and A. C. Doherty, Phys. Rev. Lett. 98, 140402 (2007).

[14] A. Peres, Found. of Phys. 29, 589 (1999).

[15] W. Dür, Phys. Rev. Lett. 87, 230402 (2001).

[16] T. Vértesi and N. Brunner, Phys. Rev. Lett. 108, 030403 (2012).
[17] R. F. Werner and M. M. Wolf, Phys. Rev. A 61, 062102 (2000).

[18] A. Acín, Phys. Rev. Lett. 88, 027901 (2001).

[19] A. Acín, V. Scarani, and M. A. Wolf, Phys. Rev. A 66, 042323 (2002).

[20] L. Masanes, Phys. Rev. Lett. 97, 050503 (2006).

[21] A. Salles, D. Cavalcanti, and A. Acín, Phys. Rev. Lett. 101, 040404 (2008).

[22] A. Salles, A. Cavalcanti, A. Acín, D. Pérez-García, and M. A. Wolf, Quant. Inf. Comp. 10, 0703 (2010).

[23] T. Moroder, J.-D. Bancal, Y.-C. Liang, M. Hofmann, and O. Gühne, Phys. Rev. Lett. 111, 030501 (2013).

[24] L. Masanes, Y.-C. Liang, and A. C. Doherty, Phys. Rev. Lett. 100, 090403 (2008).

[25] F. Buscemi, Phys. Rev. Lett. 108, 200401 (2012).

[26] M. A. Pusey, Phys. Rev. A 88, 032313 (2013).

[27] P. Skrzypczyk, M. Navascues, and D. Cavalcanti, arXiv:1311.4590.

[28] W. K. W. Lane P. Hughston, Richard Jozsa, Phys. Lett. A 183, 14 (1993).

[29] R. F. Werner, Phys. Rev. A 40, 4277 (1989).

[30] J. F. Clauser, M. A. Horne, A. Shimony, and R. A. Holt, Phys. Rev. Lett. 23, 880 (1969).

[31] B. Terhal, Phys. Lett. A 271, 319 (2000).

[32] J. Löfberg, in Proceedings of the CACSD Conference (Taipei, Taiwan, 2004), p. 284.

[33] J. F. Sturm, Optimization Methods and Software 17, 1105 (2002).

[34] O. Gühne, P. Hyllus, O. Gittsovich, and J. Eisert, Phys. Rev. Lett. 99, 130504 (2007).

[35] A. C. Doherty, P. A. Parrilo, and F. M. Spedalieri, Phys. Rev. A 69, 022308 (2004).

[36] B. M. Terhal, A. C. Doherty, and D. Schwab, Phys. Rev. Lett. 90, 157903 (2003).

[37] S. Bäuml, M. Christandl, K. Horodecki, and A. Winter, arXiv:1402.5927.

[38] T. Vertesi and N. Brunner, arXiv:1405.4502. 